\title{
A SIMPLE INTERFEROMETRIC METHOD TO MEASURE THE CALIBRATION FACTOR AND DISPLACEMENT AMPLIFICATION IN PIEZOELECTRIC FLEXTENSIONAL ACTUATORS
}

\author{
Francisco de Assis Andrade Barbosa* \\ kikoandrade83@hotmail.com \\ Ricardo Tokio Higuti* \\ tokioddee.feis.unesp.br
}

\author{
Gilder Nader ${ }^{\dagger}$ \\ gilder.nader@gmail.com \\ Cláudio Kitano* \\ kitano@dee.feis.unesp.br
}

\section{Emílio Carlos Nelli Silva ${ }^{\ddagger}$ \\ ecnsilva@usp.br}

\begin{abstract}
* Departamento de Engenharia Elétrica - Unesp
Avenida Brasil, 56, CEP 15385-000, Ilha Solteira, SP
\end{abstract}

${ }^{\dagger}$ Instituto de Pesquisas Tecnológicas, Centro de Metrologia de Fluidos

Rua Prof. Almeida Prado, 532, Cidade Universitária, CEP 05508-901 - São Paulo, SP

${ }^{\ddagger}$ Departamento de Mecatrônica e de Sistemas Mecânicos - EPUSP

Avenida Prof. Mello Moraes, 2231, CEP 05508-900, São Paulo, SP

\begin{abstract}
Laser interferometry is a well-established technique for the characterization of piezoelectric actuators. In this work, by using a low cost Michelson interferometer, the measurement of the calibration factor and the displacement amplification of a novel piezoelectric flextensional actuator (PFA), designed by using the topology optimization method, is performed. A simple method, based on small phase modulation depth when the PFA is driven by a triangular waveform, allows the absolute interferometer calibration. The free-displacement of the PFA for various drive voltages is measured and the displacement amplification is determined. The linearity and frequency response of the PFA are evaluated up to $20 \mathrm{kHz}$.
\end{abstract}

KEYWORDS: Interferometers, Displacement measurement, Piezoelectric flextensional actuators, PZT ceramics.

Artigo submetido em 13/08/2009 (Id.: 01024)

Revisado em 17/03/2010, 02/08/2010

Aceito sob recomendação do Editor Associado Prof. Sebastian Yuri Cavalcanti Catunda

\section{RESUMO}

Um método interferométrico simples para mensurar o fator de calibração e a amplificação do deslocamento em atuadores piezoelétricos flextensionais

A interferometria laser constitui uma técnica consagrada para caracterizar atuadores piezoelétricos. Neste trabalho, utilizase um interferômetro de Michelson de baixo custo para mensurar o fator de calibração e a amplificação de deslocamento de um novo atuador piezoelétrico flextensional (APF), projetado através da técnica de otimização topológica. Um método simples, baseado na excitação do APF com sinal triangular e com baixa profundidade de modulação de fase óptica, permite realizar a calibração absoluta do interferômetro. Deslocamentos do APF para várias tensões de excitação são mensurados e a amplificação do deslocamento da piezocerâmica é determinada. A linearidade e a resposta em frequência do APF, até a frequência de $20 \mathrm{kHz}$, são avaliadas.

PALAVRAS-CHAVE: Interferômetros, Medição de deslocamento, Atuador piezoelétrico flextensional, Cerâmica PZT. 


\section{INTRODUCTION}

Piezoelectric ceramics such as PZT can convert electrical energy to mechanical form (Uchino, 2000). As the PZT drive voltage changes, the piezo element changes its dimensions. Because of the free strain or displacement (in plane: $d_{31}$; out of plane: $d_{33}$ ) of the piezoceramics is small, they generally cannot be used directly as actuators in their raw form, unless high drive voltage levels are employed; consequently, amplification is required. Piezoelectric ceramic actuators usually employ mechanical amplifiers to convert the small induced strain of the ceramic material to a large output displacement, which can be used for practical applications. Compliant mechanisms are generally used as mechanical displacement amplifiers to prevent displacement losses that can occur in conventional pin-jointed mechanisms (Carbonari et al., 2007).

The use of piezoelectric actuators is increasing in various novel applications such as optical instruments (lasers and interferometers), accurate positioning in semiconductor chips manufacturing, smart composite structures, scanning probe microscopy, micromanipulation tools (needles or micro pipettes) applied to cell manipulation, and others (Niezrecki et al., 2001; Bouchilloux et al., 2004; De Abreu et al., 2004; Putra et al., 2007). They can yield large force generation, sub-millisecond response, no magnetic fields, extremely low steady-state power consumption, etc. One kind of this transducer is the piezoelectric flextensional actuator (PFA), which consists of three parts: the metal caps or shell, the active piezoelectric element, and an epoxy adhesive. Two examples are given by the moonie and the cymbal PFAs (Newnham et al., 1993; Dogan et al., 1996). PFAs have been developed in the past by using analytical models that were complemented by experimental observations in the case of simple structures, or by the finite-element method (FEM) in the case of more complex structures. The topology choice was usually based on the physical intuition of the problem.

Recently, novel models of optimized PFAs have been designed using the topology optimization technique, a computational design method that combines FEM and an optimization algorithm (Silva and Kikuchi, 2000; Silva et al., 2003). A schematic of a such PFA is shown in figure 1: the caps convert and amplify the small radial displacement and vibration velocity of the piezoceramic into a much larger axial displacement and vibration velocity normal to the surface of the caps (Marçal et al., 2007).

In this work, a prototype based on these novel flextensional piezoactuators was manufactured and analyzed. This PFA was designed with the aim to be employed, in a future work, as a mechanical controller in the reference arm of a highsensitive optical interferometer proposed to measure time-

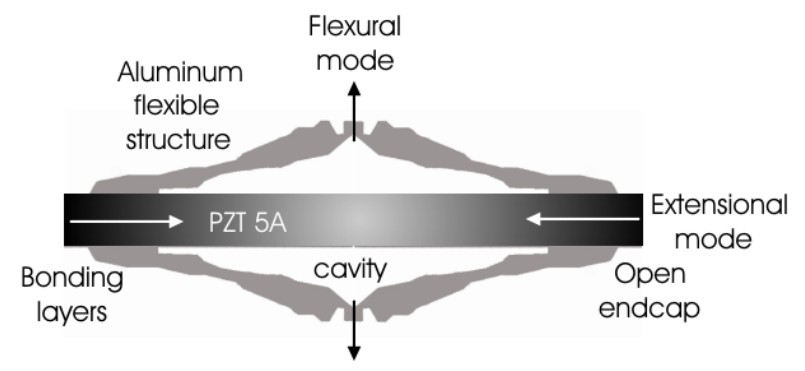

Figure 1: Schematic of the piezoelectric flextensional actuator (Marçal et al., 2007).

varying displacements with arbitrary shape in sub-angström ranges. For the purpose of mechanically stabilizing this interferometer, it was specified that the PFA should provide amplitude displacements ranging from 100 to $200 \mathrm{~nm}$, with frequency bandwidth corresponding to $3.5 \mathrm{kHz}$ at least, and maximum drive voltage equal to $150 \mathrm{~V}_{\text {peak }}$. Accordingly, in order to characterize the PFA it is necessary to measure displacements down to the nanometric scale. An important measure of the performance of the PFA mechanism is its stroke amplification or geometric advantage. The no-load amplification corresponds to the ratio of free-displacement of output-point of the PFA compliant mechanism and freedisplacement of the piezoceramic itself.

Optical interferometry provides a convenient technique for measuring microscopic displacements (Pisani, 2009; Bitou, 2009), especially those large enough (larger than $2000 \mathrm{~nm}$ ) for fringe counting techniques to be used (Wylde and Hubbard, 1999). However, for displacements that are only a small fraction of a light wavelength (such as in this work), fringe counting techniques are no more adequate, because of systematic errors involved in the method. In addition, since fluctuations in ambient temperature and pressure randomly affect the path difference between the two beams of the interferometer, the detected signal undergoes fading (Xie et al., 2009). The heterodyne and active homodyne approaches offer interesting ways to eliminate signal fading. Nevertheless, the heterodyne method is not always attractive because of the disadvantages associated with the incorporation of an optical frequency shifter (Topcu et al., 2003) or a Zeeman laser (Yokoyama et al., 2001). The signal fading may also be overcome by active stabilization schemes that maintain the phase difference between the interferometer arms at a constant value of $90^{\circ}$ (Jackson et al., 1980). Feedback can be applied directly to the interferometer though a piezoelectric transducer cylinder, located, for example, in the reference arm of an optical fiber interferometer and driven by an integrated error signal fed back from a photodetector at the interferometer output. The disadvantage of such schemes is that a complex electronic reset circuitry is necessary, and this in- 
troduces noise into the detection system. Methods of spectrum analysis of the interferometric output can also be used to measure displacements by simple passive phase-detection schemes. The $J_{1} / J_{2}$ and $J_{1} / J_{3}$ techniques were developed to be applied with a homodyne Michelson interferometer to measure the vibrational displacement ranging from 26 up to $600 \mathrm{~nm}$ at sonic and ultrasonic frequencies (Deferrari et al., 1967). However, these methods require adjusting the reference mirror of the interferometer to maximize either the fundamental or the second harmonic of the photodetected signal. In addition, they require inversion of Bessel function relationships to determine the vibration amplitudes and also problems with phase ambiguity arises, which make difficult the correct demodulation of the intended signals. On the other hand, the technique named $J_{1} \ldots J_{4}$ method provides direct, self-consistent, and linear phase readout without using any feedback or phase-bias (Sudarshanam and Srinivasan, 1989). Recently, this method has been applied to nanometric displacement measurements in PFAs by using a Michelson interferometer (Marçal et al., 2007). This technique employs well-known Bessel functions recurrence relations to determine the optical phase modulation index, and the phase measurement is unaffected by fading. However, this method of spectrum analysis has poor phase demodulation dynamic range and the time-varying displacement of the vibrating transducer face should be a pure sinusoidal function.

The system setup used in this work is a very simple, low cost and precise one, which enables the measurement of the nanometric displacements of a PFA that will be coupled to a mirror in a future assembly, functioning in another closedloop ultra sensitive interferometer as an optical phase controller. It corresponds to an homodyne Michelson interferometer operating under small phase modulation depth, and needs neither critical electronics nor complex optical parts. Additionally, it is possible to measure arbitrary time-varying displacement functions. However, the operation under small phase modulation depth is not a self-consistent approach, and a calibration factor, used to convert the photodetected voltage into nanometers, must be determined. In practice, as the PFA structure is quite complex, an accurated interferometer calibration factor cannot be analytically obtained, and hence an experimental procedure must be established to measure it. In this work, a simple method that uses a triangular waveform voltage to drive the PFA is discussed, in order to calibrate the interferometer. The paper presents the theory of operation, the layout of the system, and experimental results obtained from the laboratory system prototype.

\section{PIEZOELECTRIC ACTUATOR DESIGN AND ASSEMBLY}

In a compliant mechanism the movement is given by the structure flexibility rather than by the presence of pins and joints. The metal caps serve as mechanical transformers for converting and amplifying the lateral nanometric displacements of the ceramic into a larger axial displacement normal to the caps. Actually, both the $d_{31}$ and $d_{33}$ coefficients of the piezoelectric ceramic contribute to the axial displacement of the composite.

Topology optimization with homogenization method is applied to the design of compliant mechanisms and composite materials (Silva and Kikuchi, 1999; Li et al., 2001). It is a computational design method that combines optimization algorithms and FEM to find the optimized topology of mechanical parts considering an objective function and some constraints. Since the mechanical part of piezoelectric actuators is actually a compliant mechanism, PFAs have also been designed by using topology optimization techniques. The performance of PFAs depends on the distribution of stiffness and flexibility in the coupling structure that is bonded to the piezoceramic, which is related to the coupling structure topology. Therefore, the problem of designing a PFA is posed as the design of a flexible structure coupled to the piezoceramic that maximizes the output displacement and generative force in some specific direction. Even though the coupling structure acts as a compliant mechanism, it must be designed while coupled to the piezoceramic, since the force applied by the piezoceramic to the coupling structure depends on the flexible structure characteristics (stiffness and mass).

Figure 2 (Silva et al., 2003) illustrates the various stages involved in topology optimization procedure to design a flextensional actuator. First, an initial design domain is defined [figure 2a)], which is limited by the device boundary conditions such as regions where there are restraints and applied loads. This domain is discretized into finite elements [figure 2b)] and it is an input to the topology optimization software. The objective of topology optimization is to determine the voids and connectivities of the structure by adding and removing material in the extended fixed domain [figure 2c)]. The finite element model domain is not changed during the optimization process. The software gives as a result an optimized material distribution in the design domain, which must be interpreted [figure 2d)], verified [figure 2e)] and manufactured [figure 2f)]. By changing coupling structure topologies, novel designs of flextensional actuators are obtained for different applications, as shown for the two structures (with the same piezoceramic) in figure 3 (Silva et al., 2003). A detailed review of modelling for displacement amplified piezoelectric actuators, the FEM using ANSYS ${ }^{\mathrm{TM}}$, and their application 
to compliant mechanism design is given in Carbonari et al. (2007), Li et al. (2001), Silva and Kikuchi (2000), and Silva and Kikuchi (1999).

Specifically in this work, the device designed by topology optimization is shown in figure 4 (the same as sketched in figure 1), and it consists essentially of a piezoceramic bonded with epoxy resin to an aluminium flexible structure manufactured by using a wire EDM (Electrical Discharge Machining). The piezoceramic (PZT-5A, American Piezoceramics, $30 \mathrm{~mm} \times 13 \mathrm{~mm} \times 3 \mathrm{~mm}$ in directions 1,2 and 3, respectively) is polarized in direction 3 and electrodes are deposited on the 1-2 plane. PFA displacements decreases away from the center of the caps, where the maximum displacement is observed, to the edge, where displacement is equal to that of PZT-5A without the endcaps. The flextensional piezoactuator is fixed to a holder (not displayed in figure) by three points, perpendicular to the displacement to be measured. Thus the actuator is free to vibrate in directions 1 and 3 .

\section{THE MICHELSON INTERFEROMETER}

A low cost Michelson interferometer is employed in the measurement of PFA displacements, as sketched in figure 5. The practical utilization of the interferometric procedure relies critically on the monocromaticity and coherence degree of the light source being used (Udd, 2006). A 50/50 beam splitter divides a coherent laser beam into two equal components: a reference beam and a sensor beam directed towards the fixed and the moving mirrors, respectively. The reflected sensor beam re-enters the beam splitter, where it is directed to a photodetector. The reflected reference beam re-enters the beam splitter and it is also focused onto the photodetector. At the photodetector (a PIN photodiode), the two beams are recombined. As the sample is displaced longitudinally, the phase of the sensor beam changes relative to the reference beam, causing a fringe pattern movement.

When a time-varying voltage (the PFA drive voltage) is fed to the actuator, the sensor beam is phased modulated by the PFA displacements and the interferometric phase shift can be written as the superposition:

$$
\Phi(t)=\phi_{0}+\phi(t)
$$

where

$$
\phi_{0}=\frac{4 \pi}{\lambda} r(t)
$$

is (in principle) the static phase shift, $\phi(t)$ is the dynamic phase shift between arms, and $t$ is the time. The term $r(t)$ corresponds to any optical path variations in the reference

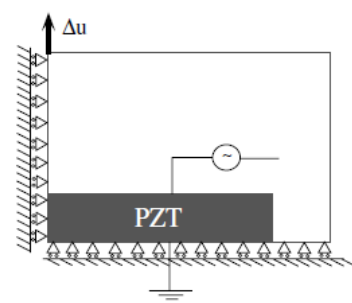

(a)

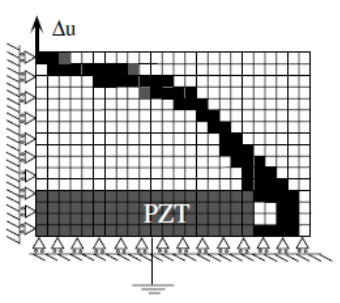

(c)

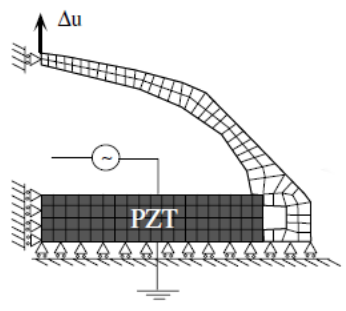

(e)

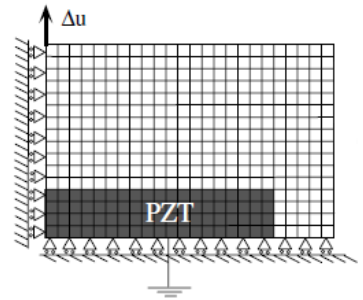

(b)

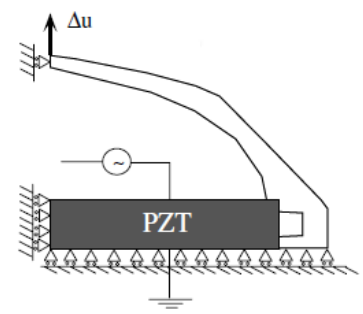

(d)

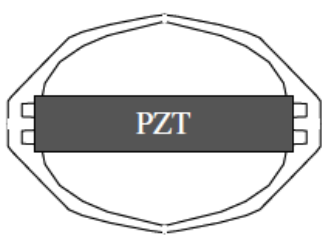

(f)
Figure 2: Topology optimization procedure (Silva et al., 2003). (a) Initial domain; (b) Discretized domain; (c) Topology obtained; (d) Interpretation; (e) Verification; (f) Manufacturing.

arm of the interferometer provided by the mirror translation stage (see figure 5), and $\lambda$ is the light wavelength in vacuum. As $\Phi(t)$ is a time-varying function, the instantaneous output voltage (or current), $v(t)$, of the photodetector is proportional to the normalized optical intensity $I / I_{0}$, where $I_{0}$ is the laser intensity $\left[\mathrm{W} / \mathrm{m}^{2}\right]$, and can be written generically as (Udd, 2006):

$$
v(t)=A[1+V \cos \Phi(t)]
$$

where $A$ is a constant depending on the laser power, photodetector responsivity, etc, and $V$ is called fringe pattern visibility $(0<V<1)$. In practical bulk interferometers, $V$ depends on the finite beam width and the coherence degree of the laser, the optical misalignment and the divergence of the gaussian beam (Deferrari et al., 1967), and consequently, it cannot be analytically determined. 


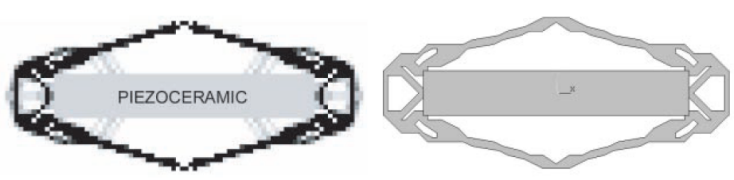

(a)

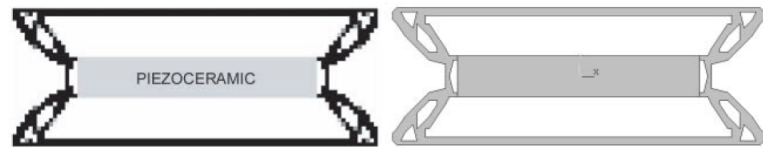

(b)

Figure 3: Topology optimization results and the corresponding interpretation (Silva et al., 2003). (a) Maximum displacement at the centre of the cap; (b) Maximum displacement at the edge of the cap.

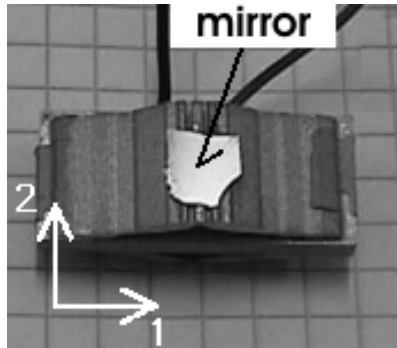

(a)

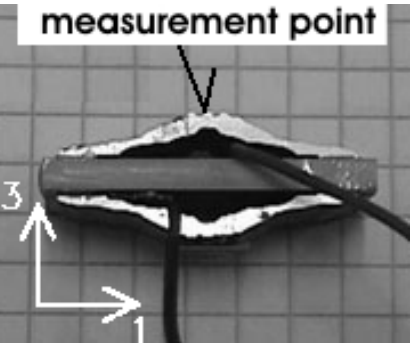

(b)
Figure 4: Prototype of manufactured piezoelectric flextensional actuator. (a) Top view; (b) Lateral view.

It can be seen from equation (3) that the interferometric output is a non-linear, sinusoidal function of the optical phase shift induced by the time-dependent signal. Hence, a direct linear measurement of signal phase from the photodetector output is not possible, unless for the case of a small phase modulation depth, which happens when $\phi(t)$ is small. The following analysis will be made under this hypothesis. In figure 6 the $I / I_{0}$ transfer function (which is proportional to the detected voltage $v$ ) is sketched relative to total phase shift $\Phi$, for the case $V=1$, as well as the small modulation depth operation under phase quadrature regime $\left(\phi_{0}=\pi / 2 \mathrm{rad}\right)$. By expanding equation (3) into a Taylor series (relative to the variable $\Phi$ ) around the point $\phi_{0}$, and selecting only the linear term, the output signal has a bias component:

$$
v_{\text {bias }}=v\left(\phi_{0}\right)=A\left(1+V \cos \phi_{0}\right) .
$$

Around point Q (see figure 6), which is associated to phase $\phi_{0}$, the slope of the transfer function, $d v /\left.d \Phi\right|_{\phi_{0}}$, is given by $\left(-A V \sin \phi_{0}\right)$. By moving the reference mirror at some very

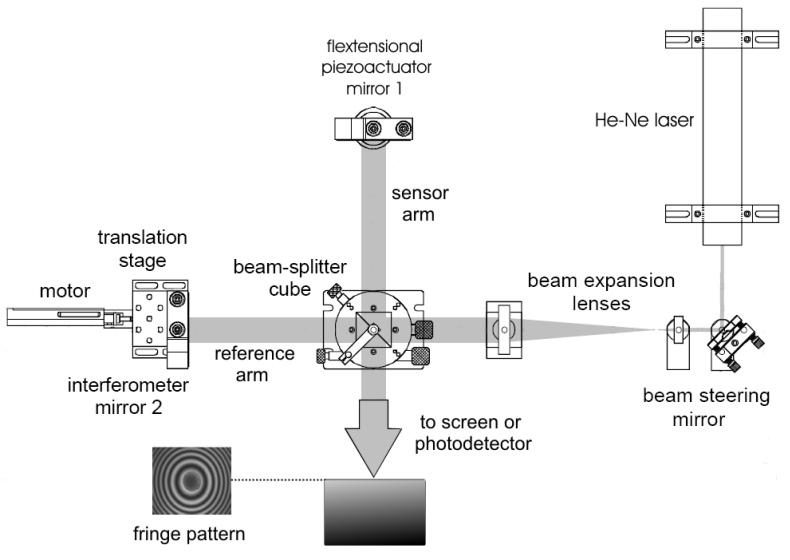

Figure 5: Homodyne Michelson interferometer used to characterize the PFA.

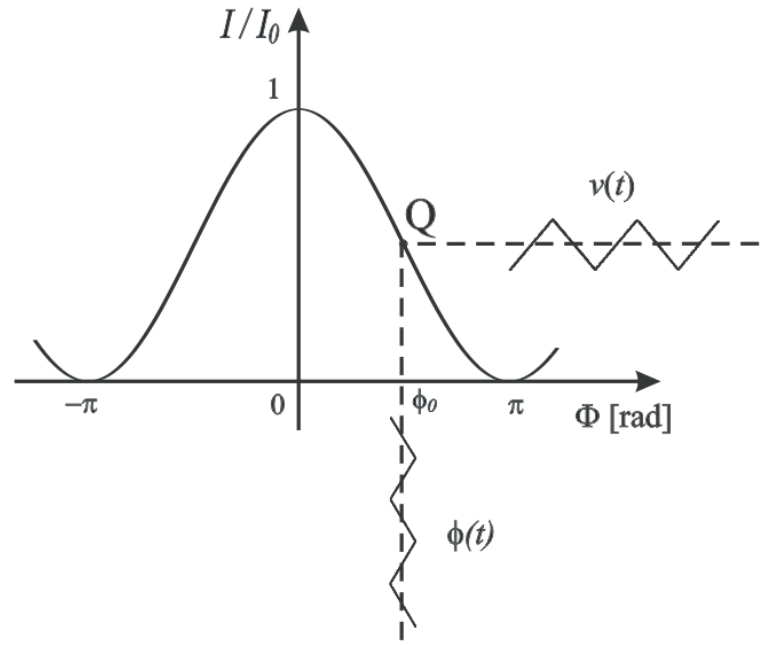

Figure 6: Interferometric transfer function and small modulation depth operation.

slow constant velocity, $\cos \phi_{0}$ and $\sin \phi_{0}$ can be made to vary from zero to unity during a measurement (but not simultaneously). The maximum slope is obtained when $\phi_{0}=\pi / 2 \mathrm{rad}$, corresponding to optimum quadrature operation, where there is a quasi-linear relationship between phase shift and output signal. Under this condition the photodetected signal is:

$$
v[\Phi(t)]=v_{\text {bias }}-A V \phi(t),
$$

which is directly proportional to the instantaneous phase shift $\phi(t)$. The $A V$ factor, in principle, is unknown, and consequently the interferometric technique requires a calibration procedure.

Interferometry constitutes a self-calibrating method, based solely on the regularity of the wavelength of the light source, 
$\lambda$ (Novikov et al., 2007). Consequently, it does not require a calibration based on comparison with a measurement reference method. In the present work the absolute calibration procedure for the interferometric measurement can be done by selecting the a.c. component from the output signal in equation (3), $v_{a c}(t)$, when the PFA is driven by a small amplitude triangular waveform and under quadrature regime, as sketched in figure 6. This condition can be established by translating the "fixed mirror" position (by using the micrometric translation stage shown in figure 5) $r(t)$, until the peak-to-peak output signal becomes maximum (Clark, 1989). As $\phi(t)$ is the corresponding a.c. phase shift, then

$$
v_{a c}(t)=A V \cos [\pi / 2+\phi(t)]=-A V \sin \phi(t) .
$$

It must be reminded that in a Michelson interferometer (Udd, 2006):

$$
\phi(t)=\frac{4 \pi}{\lambda} \Delta L(t)
$$

where $\Delta L(t)$ is the instantaneous displacement of the PFA. Next (however, still under phase quadrature operation), the PFA drive voltage is increased until $\phi(t)$ reaches the peak value corresponding to $X_{\text {peak }}=\pi / 2 \mathrm{rad}$. At this condition, the interferometric output signal becomes perfectly sinusoidal, as observed the simulation shown in figure 7a). Right after this point (which should not be exceeded in the calibration process) the output signal will present a small dip around the peak, as it can be seen in figure $7 \mathrm{~b}$ ).

Based on the behaviour of the output interferometric signal, when the phase shift between their arms is a triangular waveform, a calibration procedure can be established. The amplitude of the output signal waveform must be gradually increased until the small dip around the peak is observed. Next, the waveform amplitude must be slightly decreased in order to obtain a pure sinusoidal output. Although this procedure has been regularly applied to determine the half-wave voltage of an optical integrated modulator (Shi et al., 2000), it has not been used in interferometric applications yet.

For $\phi(t)$ adjusted exactly to $X_{\text {peak }}=\pi / 2 \mathrm{rad}$, the a.c. photodetected signal [equation (6)] reaches the maximum: $V_{\max }=-A V \sin X_{\text {peak }}$, in which $V_{\max }$ can be experimentally measured by acquiring the detected signal using an oscilloscope. By following this procedure the unknown $A V$ can be determined. The voltage $V_{\max }$ constitutes the interferometer calibration factor and need to be measured only once. Consequently, once $V_{\max }$ has been measured, and under small modulation depth condition, equation (6) can also be written as:

$$
v_{a c}(t)=V_{\max } \sin \phi(t) \cong V_{\max } \phi(t)=V_{\max } \frac{4 \pi}{\lambda} \Delta L(t)
$$

where equation (7) was used. Finally, the PFA peak displacement can be determined from equation (8) by using

$$
\Delta L_{\text {peak }}=\frac{\lambda}{4 \pi} \frac{v_{\text {peak }}}{V_{\max }},
$$

where $v_{\text {peak }}$ is the peak voltage value of $v_{a c}(t)$ under the $X_{\text {peak }}<\pi / 2$ rad condition (in other words, under small phase modulation index as shown in figure 6). According to equation (9), from now on all that is needed to measure displacements is the peak value of the a.c. output signal, while the interferometer operates under phase quadrature. However, if this last condition $\left(\phi_{0}=\pi / 2 \mathrm{rad}\right)$ is not satisfied due to experimental difficulties in adjusting the translation stage because of fading, it can be used a motorized translation stage coupled to the reference mirror and driven by a sawtooth waveform voltage. This drive voltage should be faster than typical fading frequencies $(>10 \mathrm{~Hz}$ ) with sufficiently high amplitude so that the system is forced to pass through the phase quadrature point. When this occurs, the a.c. peak-to peak output signal necessarily goes to a maximum and remains contant during a time interval sufficiently long to allow a reliable data acquisition (Clark, 1989).

\section{EXPERIMENTAL RESULTS}

The interferometric setup sketched in figure 5 was built using a $5 \mathrm{~mW}$ He-Ne laser (Newport, $\lambda=632.8 \mathrm{~nm}$ ) and a 50/50 ratio beam-splitter. The overall experimental setup is shown in figure 8 .

The PIN photodiode (Siemens, BPX65) output was amplified and the signal was digitized by an oscilloscope (Tektronix TDS2022) with 8 bits vertical resolution. The sampling rate was $1 \mathrm{MHz}$ and 2500 points were digitized for each waveform. The acquired signals were windowed with a Hamming window and the harmonic magnitudes were measured after an FFT routine in Matlab. As the maximum signal harmonics were around $1500 \mathrm{~Hz}$ and the higher harmonics have lower magnitudes, it was not necessary to use an anti-aliasing filter. Accordingly to Silva et al. (2003), and Silva and Kikuchi (1999), these PFAs are originally designed to be used in static or quasi-static mode (that is, below its first resonance frequency), thus the displacements and amplifications are determined by considering low drive voltage frequencies.

As a reflecting surface is necessary to perform the interferometric measurements, and due to the difficulties to polish the irregular actuator surface to an optical degree, a $200 \mu \mathrm{m}$ thickness mirror, obtained by aluminium vaporization over a 

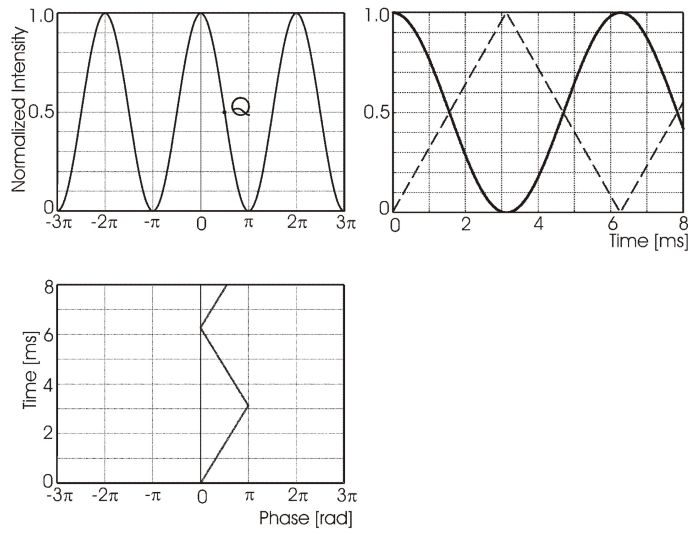

(a)
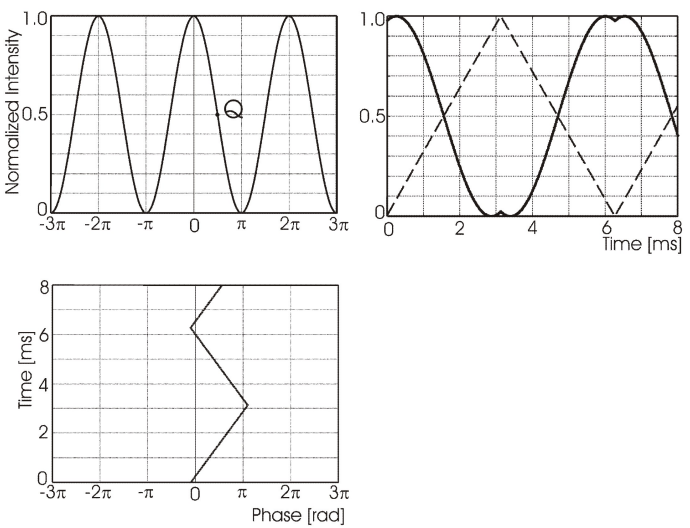

(b)

Figure 7: Principle of the calibration procedure for the interferometer under small phase modulation depth operation. The input signal (lower left) is the phase shift (proportional to PFA drive voltage), biased at quadrature condition (point $Q$ on the transfer curve, upper left), and the output signal (upper right) is the photodetected voltage. (a) Simulated output for drive voltage corresponding to $X_{\text {peak }}=\pi / 2$ rad; (b) For $X_{\text {peak }}$ right after $\pi / 2$ rad.

glass plate, was bonded to the actuator surface with epoxy resin (see figure 4). As the epoxy stiffness is of the order of $10^{10} \mathrm{~N} / \mathrm{m}^{2}$, the effect of an additional oscillator, constituted by the mirror and epoxy resin, is minimized (Silva et al., 2003). No additional resonance frequencies were experimentally observed by bonding this thin mirror to the PFA.

A practical requirement of the optical interferometry is the mechanical stability of all components of the measuring system. Free-space interferometry based on air propagation of optical beams is affected by alterations in the air environmental conditions. When air pressure, temperature or humidity change, the air refractive index will also change and so the speed of light and the light wavelength. Ideally, once $r(t)$

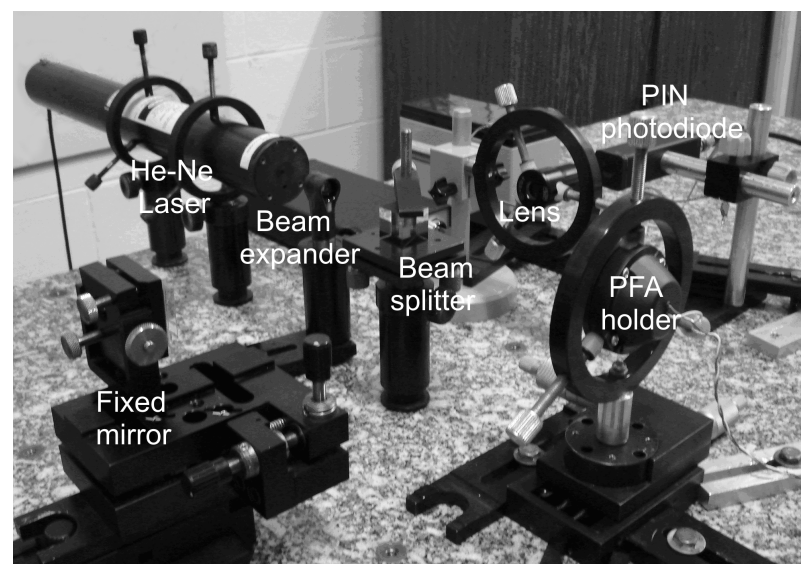

Figure 8: The overall experimental setup: bulk Michelsontype interferometer.

is adjusted so that $\phi_{0}=\pi / 2 \mathrm{rad}$, the phase $\phi_{0}$ should remain constant. However, in practice it suffers from fading, that is, it can fluctuate in a wide range and during brief periods of time due to random drifts, induced by environment perturbations (Marçal et al., 2007; Udd, 2006). Because of this, prior to measurements a 2-hour warm-up time was allowed for the system to reduce the phase drift. In addition, measurements were carried out quickly to minimize the influence of any temperature change on the system, and the assembly was mounted over a granite optical table in order to damp spurious mechanical vibrations.

Initially, for calibrating the interferometer, the PFA was driven by triangular waveforms with amplitudes ranging from 2.5 to $58 \mathrm{~V}_{\text {peak }}$ at $125 \mathrm{~Hz}$. This frequency is high enough to avoid the low frequency environmental vibrations but low enough to be considered in quasi-static regime. Examples of digitized photodetected waveforms are shown in figure 9. In figure 9a) the interferometer is operating under small modulation depth. When the drive voltage reaches 39 $\mathrm{V}_{\text {peak }}$ the a.c. output signal becomes fairly sinusoidal [see figure 9b)], with $V_{\max }=64 \mathrm{mV}_{\text {peak }}$. Henceforth, the displacements can be measured by applying equation (9). As shown in figure $9 \mathrm{c}$ ), when the drive voltage is $58 \mathrm{~V}_{\text {peak }}$, a dip is present in the waveform.

Additionally, in order to increase the accuracy in the measurement of the calibration factor $V_{\max }$, it is proposed to evaluate the harmonic distortion in the photodetected signal, for both conditions $X_{\text {peak }}=\pi / 2 \mathrm{rad}$ and $\phi_{0}=\pi / 2 \mathrm{rad}$. In the ideal case, the output signal should be a pure sinusoid. Figure 10 shows the magnitude spectrum of the signal represented in figure $9 \mathrm{~b})$. There is a fundamental frequency at 125 $\mathrm{Hz}$, and harmonics at $250 \mathrm{~Hz}(-34 \mathrm{~dB})$ and $375 \mathrm{~Hz}(-31 \mathrm{~dB})$, resulting in a harmonic distortion around $4 \%$. In these conditions, the detected signal can be considered a good sinusoid. 

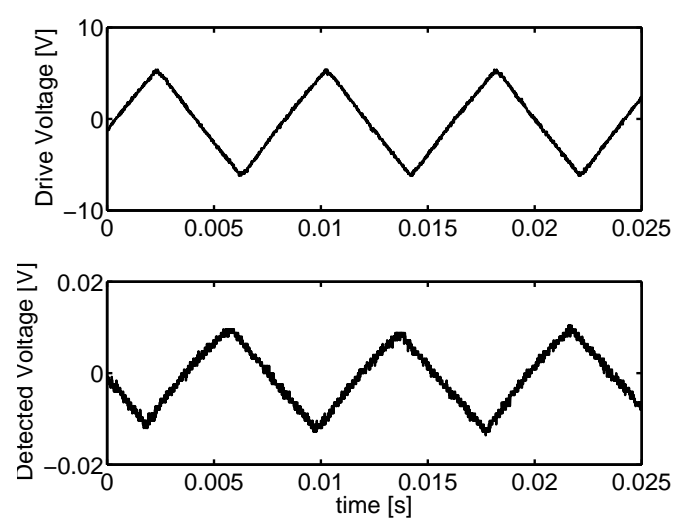

(a)
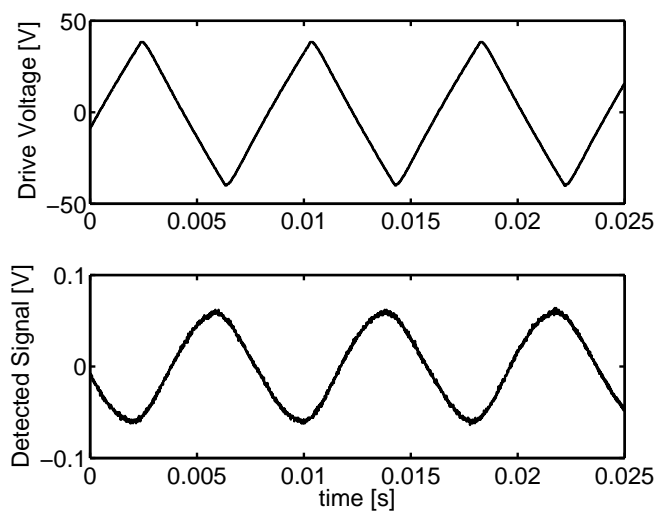

(b)
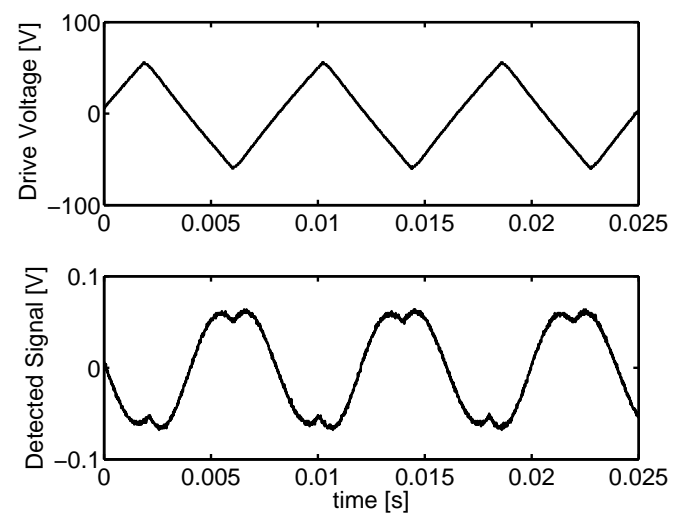

(c)

Figure 9: Photodetected signals (a.c. coupling) used for calibrating the interferometric results. In all the cases the upper signal corresponds to PFA drive voltage and the lower one is the photodetected signal. (a) For triangular waveform, 5.8 $\mathrm{V}_{\text {peak }}$ drive voltage at $125 \mathrm{~Hz}$; (b) For $39 \mathrm{~V}_{\text {peak }}$ at $125 \mathrm{~Hz}$; (c) For $58 \mathrm{~V}_{\text {peak }}$ at $125 \mathrm{~Hz}$.

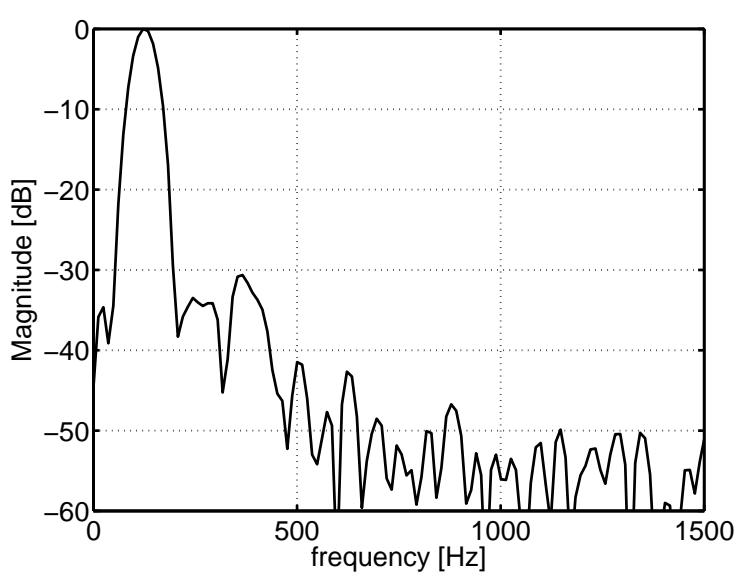

Figure 10: Spectrum of the photodetected signal when the PFA is driven with $39 \mathrm{~V}_{\text {peak }}$ at $125 \mathrm{~Hz}$ and under quadrature regime.

Figure 11 shows the displacement amplitudes at the cap center of the composite actuator for different triangular drive voltages at the fundamental frequency of $125 \mathrm{~Hz}$. The curve presents a linear response over the displacement range considered, and the least squares fit is shown with a $0.8 \mathrm{~nm}$ standard deviation. The slope of the curve is $1.36 \mathrm{~nm} / \mathrm{V}_{\text {peak }}$. Thus, the PFA calibration factor at $125 \mathrm{~Hz}$ is equal to 1.36 $\mathrm{nm} / \mathrm{V}$. The displacement amplification rate is obtained for each frequency by dividing the displacement generated by the actuator and the displacement generated by the piezoceramic in the 1-direction $\left(d_{31}\right)$ and 3-direction $\left(d_{33}\right)$, considering the same drive voltage. By using the results obtained in Marçal et al. (2007), this amplification can also be measured.

Piezoceramic materials such as PZT-5A have its dielectric, piezoelectric and mechanical properties generally given by manufacturer manuals with up to $5 \%$ difference from manufacturer to manufacturer. However, if these values are actually measured, deviations up to $20 \%$ can be obtained. Therefore, it is important to determine experimentally the strain coefficients of piezoceramics. Based on the work of Marçal et al. (2007), piezoceramic transducer relevant data were measured by using a commercial MTI-2000 fotonic sensor $^{\mathrm{TM}}$ (MTI-2020R, $90 \mathrm{kHz}$ frequency response range, with 101 and $1457 \mathrm{~nm}$ linear ranges, and 30 and $250 \mathrm{~nm}$ resolutions, in its 1- and 2-operation ranges, respectively) operating with a $1 \mathrm{~Hz}$ triangular waveform. The following values were obtained for the piezoelectric coefficients: $d_{31}=-133$ $\mathrm{pm} / \mathrm{V}$ and $d_{33}=287 \mathrm{pm} / \mathrm{V}$.

According to Marçal et al. (2007), the PZT-5A piezoceramic used in this work was also modelled by FEM and experimentally tested by measuring its electrical admittance up to 60 $\mathrm{kHz}$. The results revealed that the first resonance frequency, 


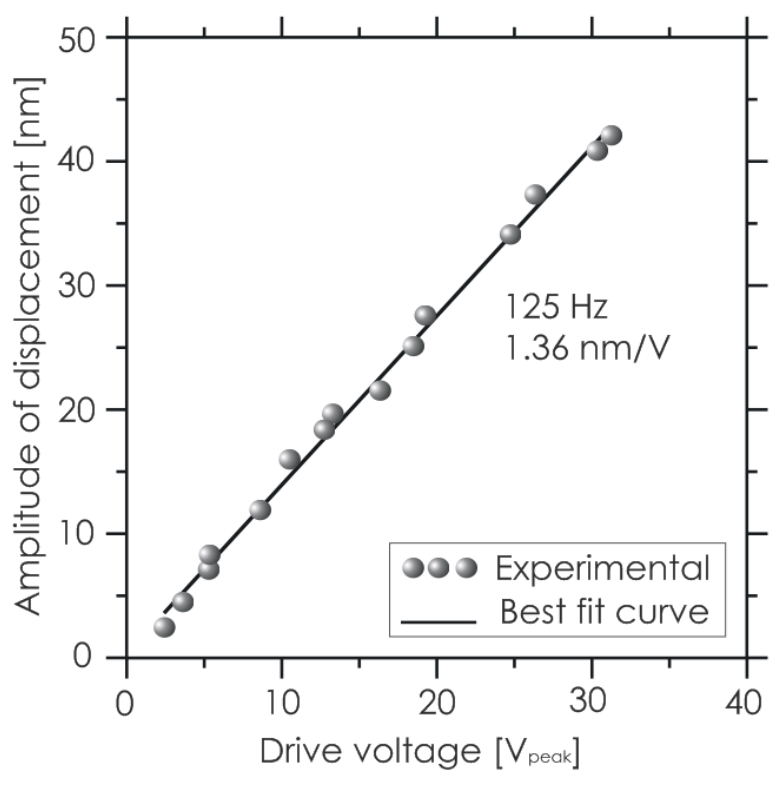

Figure 11: Displacement amplitudes as a function of the drive voltage (triangular waveform at $125 \mathrm{~Hz}$ ) applied to the PFA. The least-square fit to this plot is also shown $(y=1.36 x+$ $0.30)$.

related to the length mode shape, occurs at $46.4 \mathrm{kHz}$. So, roughly speaking, the piezoceramic behaves linearly up to this frequency, and the $d_{31}$ and $d_{33}$ coefficients (measured at $1 \mathrm{~Hz}$ ) should not vary considerably with frequency in this range. Because of this, in the present work, it will be assumed that values of $d_{31}$ and $d_{33}$ at $1 \mathrm{~Hz}$ and $125 \mathrm{~Hz}$ remain approximately constant. Next, the slopes of the PFA displacement curves obtained with MTI-2000 and ANSYS were determined and displayed in table 1 , as well as that obtained from figure 11. By using the above values of $d_{31}$ and $d_{33}$ coefficients, the amplification measurements (in directions 1 and 3) are obtained by the interferometric, MTI-2000 and ANSYS methods.

As can be seen from table 1, although the amplification results measured with the interferometer and the MTI-2000 are close ( $1 \%$ difference), the amplification obtained from ANSYS is very different (39\% higher relative to interferometer results). This is not a rule because ANSYS always provided excellent results when applied to PFA with closed endcap structure (Silva et al., 2003), like those shown in figure 3. In closed endcap actuators the displacement transmission from piezoceramic to the flexible structure involves two types of actions: a pressure mechanism between the ends of the piezoceramic and the flexible structure and, possibly, a shear mechanism between the upper and lower piezoceramics electrodes and the flexible structure. The presence of the epoxy layer between the piezoceramic and the flexible structure, the existence of inhomogeneities in bonding thickness
Table 1: PFA no-load amplification measurement.

\begin{tabular}{lccc}
\hline \hline Method & $\begin{array}{c}\text { Slope } \\
(\mathbf{n m} / \mathbf{V})\end{array}$ & $\begin{array}{c}\text { Amplification } \\
\mathbf{3 3}\end{array}$ & $\begin{array}{c}\text { Amplification } \\
\mathbf{3 1}\end{array}$ \\
\hline This work & 1.36 & 9.50 & 2.04 \\
MTI-2000 & 1.35 & 9.40 & 2.03 \\
ANSYS & 1.90 & 13.2 & 2.86 \\
\hline
\end{tabular}

generated by surface roughness as well as the appearance of local meniscus and lacks of adhesive, may introduce some non-linear behavior in this displacement transmission mechanism (Ochoa et al., 2007). The pressure mechanism tends to be less affected by the epoxy layer non-linear behavior than the shear mechanism. In summary, the closed structure is not as significantly affected as the open ended PFA (as the one used in this work) by the epoxy layer non-linear behavior.

A fast response time is another important criterion for the PFA and it can be defined as the time to achieve the quick and precise response of the actuator without overshoot and ringing. The mechanical resonance of the system limits the practical actuating range. When arbitrary signals are applied to the PFA, as in practical micrometric or nanometric positioners, higher resonance frequencies can be excited, and the linearity between control signal and the corresponding displacement is lost. Positioning actuators should be used only in the linear range of their spectra, well below any resonance frequency. Such a phenomenon is usually known as tracking error (Bergander et al., 2003; Marçal et al., 2005). This is why it is important to know the piezoactuator frequency response, in order to determine (and avoid) its resonance frequencies.

Figure 12 shows the experimental frequency response of the PFA measured using interferometer, in terms of the amplitude displacement normalized by the input voltage, $(\mathrm{nm} / \mathrm{V})$. The gain is fairly constant up to $4 \mathrm{kHz}$, equal to $1.4 \mathrm{~nm} / \mathrm{V}$ approximately. The first two resonance frequencies are found at 4.6 and $15 \mathrm{kHz}$ (actually there are a large number of higher resonance frequencies, but they are not shown in figure), far from the PFA typical operation band. It should be emphasized that simulations with ANSYS were not able to detect these two resonances; the first previewed resonance occurred at $20 \mathrm{kHz}$. Again, this probably happens due to difficulties in modelling the open ended PFA and the non-linear properties of the epoxy resin.

\section{CONCLUSIONS}

In the present paper the calibration factor of a novel flextensional piezoactuator, previously designed by using the topology optimization method developed by Silva and Kikuchi (1999), and Silva et al. (2003), was experimentally deter- 


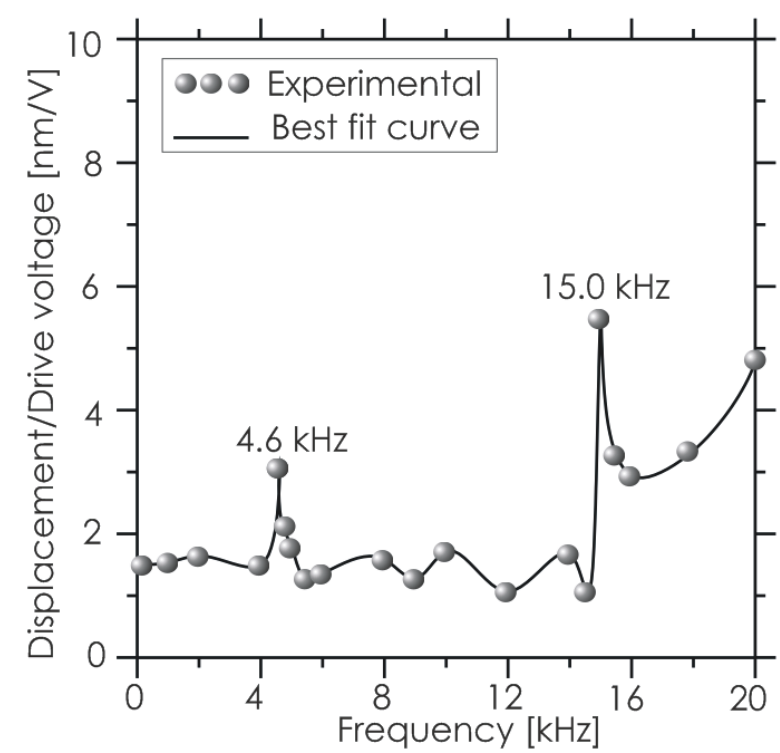

Figure 12: Interferometric transfer function and small modulation depth operation.

mined by the measurement of nanometric displacements using a low cost homodyne Michelson interferometer. In the theoretical analysis the hypothesis of $V=1$ and small modulation depth operation were considered. This measurement method is very reliable once the information is based on light phase modulation, rather than light intensity as happens for the MTI-2000 optical fiber sensor. This makes the interferometer calibration procedure independent of any external standard, only depending on the knowledge of light wavelength. As the best resolution of MTI-2000 is $30 \mathrm{~nm}$, the PFA was excited with amplitudes as high as $500 \mathrm{~V}_{\text {peak }}$ at the frequency of $1 \mathrm{~Hz}$. On the other side, by using the interferometer, the PFA was excited with amplitudes below $58.0 \mathrm{~V}_{\text {peak }}$, measuring the calibration factor of $1.36 \mathrm{~nm} / \mathrm{V}$ with standard deviation of only $0.8 \mathrm{~nm}$. In addition, unlike the light intensity modulation, the phase modulation provides excellent rejection of background light.

Together with the MTI-2000 results (Marçal et al., 2007), it can be concluded that the PFA performance was linear up to $200 \mathrm{~V}_{\text {peak }}$, with displacement amplification of 9.5 (associated to $d_{33}$ ) and 2.04 (associated to $d_{31}$ ) in 3 and 1 directions, respectively. The PFA has a reasonably flat response below 4 $\mathrm{kHz}$, where the gain is approximately constant, and the first significant resonance occurs at $4.6 \mathrm{kHz}$. Consequently, a 220 $\mathrm{nm}$ displacement can be obtained when $150 \mathrm{~V}_{\text {peak }}$ voltage is applied to this PFA. In conclusion, the PFA designed by using topology optimization method satisfied the required specification to implement optical phase controllers to be used, in closed loop interferometer applications in future works.

\section{ACKNOWLEDGEMENTS}

The authors would like to thank the Brazilian sponsor agencies FAPESP and CAPES.

\section{REFERENCES}

Bergander, A., Driesen, W., Varidel, T. and Breguet, J. M. (2003). Development in miniature manipulators for application in biology and nanotechnologies, Proc. of the Microrobotics for Biomanipulation Workshop, IEEE/RSJ Int. Conf. on Intelligent Robots and Systems (IROS 03), Las Vegas, USA, pp. 11-35.

Bitou, Y. (2009). High-accuracy displacement metrology and control using a dual fabry-perot cavity with optical frequency comb generator, Precision Engineering 33(2): 187-193.

Bouchilloux, P., Claeyssen, F. and LeLetty, R. (2004). Amplified piezoelectric actuators: From aerospace to underwater applications, Proc. of the SPIE, Smart Structures and Materials 2004: Industrial and Commercial Applications of Smart Structures Technologies, San Diego, USA, pp. 143-154.

Carbonari, R. C., Silva, E. C. N. and Nishiwaki, S. (2007). Optimum placement of material in piezoactuator design, Smart Materials and Structures 16(1): 207-220.

Clark, N. H. (1989). An interferometric method to measure oscillatory displacements, Metrologia 26(2): 127-133.

De Abreu, G. L. C. M., Ribeiro, J. F. and Steffen Jr., V. (2004). Finite element modelling of a plate with localized piezoelectric sensors and actuators, Journal of the Brazilian Society of Mechanical Sciences and Engineering XXVI(2): 117-128.

Deferrari, H. A., Darby, R. A. and Andrews, F. A. (1967). Vibrational displacement and mode-shape measurement by a laser interferometer, The Journal of the Acoustical Society of America 42(5): 982-990.

Dogan, A., Fernandez, J. F., Uchino, K. and Newnham, R. E. (1996). The cymball electromechanical actuator, Proc. of the Tenth IEEE International Symposium on Applications of Ferroelectrics, ISAF 96, New Jersey, USA, pp. 213-216.

Jackson, D. A., Priest, R., Dandridge, A. and Tveten, A. B. (1980). Elimination of drift in a single-mode optical fiber interferometer using a piezoelectrically stretched coiled fiber, Applied Optics 19(17): 2923-2929.

Li, Y., Xin, X., Kikuchi, N. and Saitou, K. (2001). Optimal shape and location of piezoelectric materials for topology optimization of flextensional actuators, Proc. of the 
2001 Genetic and Evolutionary Computation Conference, GECCO-2001, San Francisco, USA, pp. 10851089.

Marçal, L. A. P., Leão, J. V. F., Nader, G., Higuti, R. T., Kitano, C. and Silva, E. C. N. (2007). Analysis of linearity and frequency response of a novel piezoelectric actuator using a homodyne interferometer and the $j_{1}-j_{4}$ method, IEEE Transactions on Instrumentation and Measurement 56(3): 954-961.

Marçal, L. A. P., Leão, J. V. F., Nader, G., Silva, E. C. N., Higuti, R. T. and Kitano, C. (2005). Dynamic analysis of a new piezoelectric flextensional actuator using the $j_{1}-j_{4}$ optical interferometric method, Proc. of Rio 2005 Internoise Environmental and Control - The 2005 Congress and Exposition on Noise Control Engineering, Rio de Janeiro, Brazil, pp. 1-10.

Newnham, R. E., Dogan, A., Xu, Q. C., Onitsuka, K., Tressler, J. and Yoshikawa, S. (1993). Flextensional moonie actuators, Proc. of the IEEE 1993 Ultrasonic Symposium, Baltimore, USA, pp. 509-513.

Niezrecki, C., Brei, D., Balakrishna, S. and Moskalik, A. (2001). Piezoelectric actuation: State of the art, The Shock and Vibration Digest 33: 269-280.

Novikov, Y. A., Ozerin, Y. V., Rakov, A. V. and Todua, P. A. (2007). Method for linear measurement in the nanometre range, Measurement Science and Technology, 18(2): 367-474.

Ochoa, P., Pons, J. L., Villegas, M. and Fernandez, J. F. (2007). Effect of bonding layer on the electromechanical response of the cymbal metal-ceramic piezocomposite, J. European Ceramic Society 27(2-3): 11431149.

Pisani, M. (2009). A homodyne Michelson interferometer with sub-picometer resolution, Measurement Science and Technology 20(8): 1-6.

Putra, A. S., Huang, S., Tan, K. K., Panda, S. K. and Lee, T. H. (2007). Design, modelling, and control of piezoelectric actuators for intracytoplasmatic sperm injection, IEEE Transactions on Control Systems Technology 15(5): 875-890.

Shi, Y., Lin, W., Olson, D. J., Bechtel, J., Zhang, H., Steier, W. H., Zhang, C. and Dalton, L. R. (2000). Electrooptic polymer modulators with $0.8 \mathrm{v}$ half-wave voltage, Applied Physics Letters 77(1): 1-3.

Silva, E. C. N. and Kikuchi, N. (1999). Design of piezoelectric transducers using topology optimization, Smart Materials and Structures 8(3): 350-364.
Silva, E. C. N. and Kikuchi, N. (2000). Topology optimization design of flextensional actuators, IEEE Transactions on Ultrasonic, Ferroelectrics, and Frequency Control 47(3): 597-605.

Silva, E. C. N., Nader, G., Shirahige, A. B. and Adamowski, J. C. (2003). Characterization of novel flextensional actuators designed by using topology optimization method, Journal of Intelligent Material Systems and Structures 14(4-5): 297-308.

Sudarshanam, V. S. and Srinivasan, K. (1989). Linear readout of dynamic phase change in a fiber-optic homodyne interferometer, Optics Letters 14(2): 140-142.

Topcu, S., Chassagne, L., Haddad, D. and Alayli, Y. (2003). Heterodyne interferometric technique for displacement control at the nanometric scale, Review of Scientific Instruments 74(11): 4876-4880.

Uchino, K. (2000). Ferroelectrics Devices, Marcel Dekker, New York.

Udd, E. (2006). Fiber Optic Sensors - An Introduction for Engineers and Scientists, John Wiley \& Sons, New York.

Wylde, J. and Hubbard, T. J. (1999). Measurement of MEMS displacements and frequencies using laser interferometer, Proc. of the 1999 IEEE Canadian Conference on Electrical and Computer Engineering, Edmonton, Canada, pp. 1680-1685.

Xie, F., Chen, X. and Zhang, L. (2009). High stability interleaved fiber Michelson interferometer for on-line precision displacement measurements, Optics and Lasers in Engineering 47(11): 1301-1306.

Yokoyama, T., Araki, T., Yokoyama, S. and Susuki, N. (2001). A subnanometer heterodyne interferometric system with improved phase sensitivity using a threelongitudinal-mode He-Ne laser, Measurement Science and Technology 12(2): 157-162. 